\title{
The Radius of Electron
}

\author{
Salah Eid \\ Abstract: \\ Suez Canal University - Faculty of Arts - Ismailia - Egypt \\ One electron inside neutron's system divides its $2.2 \mathrm{Mev}$ energy between its proton in this system and another \\ proton forming deuteron nucleus, and two electrons with negative and positive charges forming gamma ray that \\ separated the neutron from the proton in deuteron nucleus experimentally. Using our universal constant U, we can \\ determine \\ 1- the radius of neutron nucleus in the first state as the basic nuclear radius in the universe according to the \\ well known historical result of Rutherford, and \\ 2- Determine, for the first time, the radius of the electron in the second state.
}

DOI: $10.7176 /$ APTA/83-07

Publication date: April $30^{\text {th }} 2020$

\section{Introduction}

The spin of the electron or its revolution around its axis as a charged particle is a very clear fact. Around 1928 Uhlenbec and Gaudsmith proposed the idea that each electron spins with an angular momentum of one half of Plank constant and carriers a magnetic moment of one Bohr magneton. ${ }^{(1)}$ When Paul Dirac derived his relativistic quantum mechanics in 1928, electron spin was an essential part of it ${ }^{(2)}$

In fact, without the electron's spin no charge will take place on it. Its spin means that it is a small rigid ball with a necessary small radius. We have a good chance for determining its radius through the combination of the electron with the positron or the electron with positive charge, the interaction between two electrons turns both of them out completely to gamma ray. We accustomed to believe that these two particles are annihilated in two rays of gamma , but no annihilation occurs in this state, the result is one entity from the two particles in gamma. As the energy of gamma separated the neutron from proton in the smallest nucleus called deuteron, and this energy was experimentally $2.2 \mathrm{Mev}=3.524 \times 10^{-13} \mathrm{~J}$. therefore we can determine the radius of the two combined particles $2 m_{e}$ in one entity. Using our unifying constant $\mathrm{U}=2.30 \times 10^{-28} \mathrm{J-m}$ extracted from Coulomb's law ${ }^{(3)}$, and dividing the result by 2 we have the radius of one electron.

1- Determining the radius of deuteron:

With $2.2 \mathrm{mev}=3.524 \times 10^{-13} \mathrm{~J}$, the energy of one electron in neutron's systems, this electron binds its proton in this system with another proton creating the deuterium nucleus called deuteron. We can determine the nuclear radius of the deuteron using our constant $U$ where the electron divides its energy between them as follows

$$
\frac{2.30 \times 10^{-28 ~ J-m}}{\frac{3524 \times 10^{-13}}{2}}=1.30 \times 10^{-15} \mathrm{~m} \text {. (4) }
$$

This is indeed the basic nuclear radius in the smallest nuclei in the universe according to the result Rutherford reached to through his well known experiment about the nuclear radius.

\section{2- Electron's radius from dissociated deuteron:}

On the other hand, the neutron had been separated from the proton in deuteron by Bethe experimentally in 1938 , he used gamma ray having the previous energy $2.2 \mathrm{Mev}=5.324 \times 10^{-13} \mathrm{~J} .^{(5)}$ As gamma ray is the result of completely interaction between two electrons of mass $2 m$ with their two equal and opposite charges, and because these two charges are the two equal and opposite halves of one circular motion in gamma ray then the radius of the two combined electrons is as follows

Therefore the radius of one electron is

$$
\frac{2.30 \times 10^{-28 J-m}}{3.524 \times 10^{-13}}=6.524 \times 10^{-16} \mathrm{~m}
$$

$$
\frac{6.524 \times 10^{-16}}{2}=3.623 \times 10^{-16} \mathrm{~m} .
$$

It is about $\frac{1}{4}$ the smallest basic nuclear range. 


\section{Conclusion:}

$2.2 \mathrm{Mev}=3.524 \times 10^{-13} \mathrm{~J}$ was the energy of gamma ray that separated one neutron from one proton forming deuteron nucleus, as gamma consists of two electrons of charges $e^{-}$and $e^{+}$, these two equal and opposite charges are the two halves of circular motion of gamma with the necessary radius $r$ for the two electrons. Now, half of this radius of both electrons forming one entity is the radius of one electron $=3.623 \times 10^{-16} \mathrm{~m}$.

\section{References:}

1- http:// www. Lorentz .org. historic, spin,ft

2- https:// en. Wikipedia, wiki. Spin

3- Eid Salah: The unifying Constant U, Advances in Physics, theories and Applications, Vol 36, 2014

4- The previous reference

5- Bethe H.A. Physical Review, February, 15,1938, vol 53 P 313-314 